San Jose State University

SJSU ScholarWorks

Faculty Publications

Electrical Engineering

2005

\title{
Modulation Identification and Carrier Recovery System for Adaptive Modulation in Satellite Communications
}

Kenta Umebayashi

University of Oulu

Robert H. Morelos-Zaragoza

San Jose State University, robert.morelos-zaragoza@sjsu.edu

Ryuji Kohno

Yokohama National University

Follow this and additional works at: https://scholarworks.sjsu.edu/ee_pub

Part of the Electrical and Computer Engineering Commons

\section{Recommended Citation}

Kenta Umebayashi, Robert H. Morelos-Zaragoza, and Ryuji Kohno. "Modulation Identification and Carrier Recovery System for Adaptive Modulation in Satellite Communications" Faculty Publications (2005). https://doi.org/10.1109/VETECS.2005.1543825

This Article is brought to you for free and open access by the Electrical Engineering at SJSU ScholarWorks. It has been accepted for inclusion in Faculty Publications by an authorized administrator of SJSU ScholarWorks. For more information, please contact scholarworks@sjsu.edu. 


\title{
Modulation Identification and Carrier Recovery System for Adaptive Modulation in Satellite Communications
}

\author{
Kenta Umebayashi*, Robert H. Morelos-Zaragoza ${ }^{\dagger}$ and Ryuji Kohno ${ }^{\ddagger}$ \\ ${ }^{*}$ Centre for Wireless Communications, University of Oulu P. O. Box 4500 FIN-90014 University of Oulu, Finland \\ Email: ume@ee.oulu.fi \\ ${ }^{\dagger}$ San Jose State University One Washington Square San Jose, CA 95192-0084 \\ Email: rmorelos@email.sjsu.edu \\ ${ }^{\ddagger}$ Yokohama National University 79-5 Tokiwadai, Hodogaya, Yokohama, Kanagawa, 240-8501, Japan. \\ Email: kohno@ynu.ac.jp
}

\begin{abstract}
We introduce the modulation identification technique implementing the multimode phase locked loop (PLL) in the satellite communication using adaptive modulation scheme which is a countermeasure against the rain attenuation. In the multimode PLL, phase lock detectors (PLDs) are used for not only phase lock, but also modulation identification. We present the sub-optimized design of the PLDs for modulation identification with respect to the throughput and show the validity of sub-optimization. In addition, by the comparison between the multimode PLL and conventional scheme in ISDB-S, we present the effectivity of the multimode PLL.
\end{abstract}

\section{INTRODUCTION}

A modulation identification technique is a challenging topic that has been studied for military, surveillance, Software Defined Radio (SDR) and non-cooperative communications. The modulation identification has played a key role in a military communication and surveillance fields. However, in the consumer applications, the efficiency of the modulation identification techniques remains unknown, therefore, this paper focus on this problem.

Wei and Mendel presented the optimum modulation classification method based on Bayes decision theory where the criterion is the minimum error-rate of the modulation classification [1]. An objective of [1] was to develop the theoretical analysis of generic Maximum Likelihood (ML) classifier for the phase and amplitude modulation schemes. In [1] and [2], they utilized the ML or Likelihood Function (LF) for the modulation identification. They have two critical problems which are the realities of assumptions and the computational complexity. [1] assumed that the synchronization is ideal and noise power is known at the receiver, however, it is unrealistic. In [2], even though, the carrier offset was considered, huge amount of computation is necessary due to the implementation of LF functions.

Based on the above problems, we have been working on multimode Phase Locked Loop (PLL) which can identify the modulation type and recover the carrier offset. The main contributions of this study are as follows. 1) We discover a sub-optimized design of Phase Lock Detectors (PLD) which are main part of modulation identification. The modulation identification error and non-modulation identification (nonMODID) are main causes deteriorating the throughput. Here non-MODID means that there is not the modulation identification result, namely, it is detected that all of the modulation types are unreliable. For this problem, the sub-optimization in terms of throughput is derived by minimizing the summation of the modulation identification error rate $(E R)$ and nonMODID rate $(N R)$. 2) We present a comparison between the multimode PLL and the conventional scheme on Integrated Services Digital Broadcasting - Satellite (ISDB-S) which is a broadcasting communication from the satellite to the earth stations on the Ku-band. This comparison shows an efficiency, i.e. the throughput, based on modulation identification regarding one of the consumer applications. There has been no study that tried to evaluate the modulation identification techniques regarding the throughput or quality of the communication.

The remainder of the paper is organized as follows. In Section II, the assumptions and system block diagram are presented. In Section III, we describe the modulation identification algorithm and in Section IV, which is a main part of this paper, we present the sub-optimized design of PLDs. Numerical results are shown in Section V, and finally, we conclude and outline the future subjects in Section VI.

\section{SYSTEM DESCRIPTION}

We assume the adaptive modulation on ISDB-S. Assumed Channel model, described in section $\mathrm{V}$, is based on the ISDB$\mathrm{S}$ standard [4] and Ku band channel model [5]-[7], where the received signal power is varied due to the rain attenuation. In this paper, we concentrate on modulation identification problem at the receiver, therefore, the modulation control in the transmitter is assumed complete. Although the receiver does not have information of which modulation type is used, the information of the available modulation types is provided. In fact, in the conventional ISDB-S system, BPSK, QPSK and 8PSK are available, and the modulation type is informed by the pilot signals corresponding to redundancy. On the other 


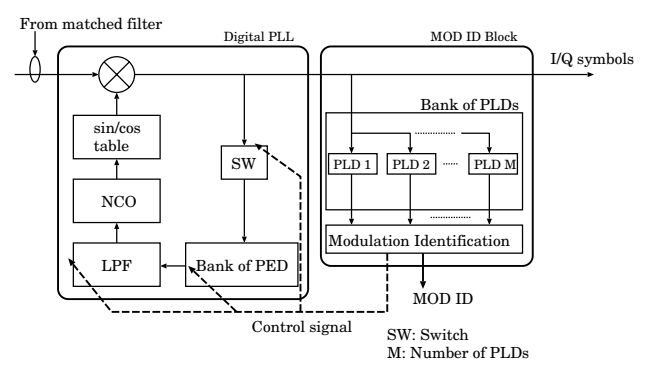

Fig. 1. System block diagram of multimode PLL

hand, in our proposal, multimode PLL has a modulation identification algorithm instead of pilot signals, hence, redundancy can be deleted. The general multimode PLL, to be hereinafter described in detail, is illustrated in Fig. 1. The $n$-th baseband received signal in the multimode PLL is given by

$$
r(n)=\exp \left[-j\left(\phi+2 \pi \Delta f T_{s} n\right)\right] s(n)+g(n),
$$

where $\phi, \Delta f, T_{s}$ and $g(t)$ are the initial phase offset, the carrier offset, the symbol interval and Additive White Gaussian Noise (AWGN), respectively. Let $\Delta f$ be small compared with the symbol rate $R_{s}=1 / T_{s}$, thus, the received signal lies within the capture range of the digital PLL. This condition is equivalent to $\Delta f T_{s}<<1$. Equivalently, this result can also be applied to single-carrier narrow-band systems, as well. Let the normalized frequency offset be equal to $\Delta f T_{s}$. In addition, $s(n)$ is the $n$-th transmitted symbol baseband signal and is given by

$$
s(n)=\exp \left[j\left(\psi_{M O D}+\frac{2 \pi C_{M O D}(n)}{C_{M O D, M A X}+1}\right)\right]+g(n),
$$

where $\psi_{M O D}$ and $C_{M O D}(n)$ are number depending on the modulation type and $M O D$ indicates the modulation type. In fact, $\psi_{B P S K}=0, \psi_{Q P S K}=\pi / 4$ and $\psi_{8 P S K}=\pi / 8$, and $C_{M O D}(n)$ is integer regarding the input bits, i.e $C_{Q P S K}(n) \in(0,1,2,3)$ and $C_{Q P S K, M A X}(n)=3$.

Carrier and phase offsets make it difficult to identify the modulation type. For this problem, we has implemented the conventional digital PLL which can cancel those offsets. However, several modules in multimode PLL have to be specified by the modulation type, and this can be conflicting. it Namely, for the offsets problem, digital PLL can solve it, however, the modulation type must be known. On the other hand, in the modulation identification problem, as mentioned above, the phase offsets make it difficult, if we do not implement any countermeasure, e.g. digital PLL. In this study, we consider not only the modulation but also identification and phase offset problems. A solution for this problem is shown as follows by explaining the procedure and configuration of the multimode PLL. As shown in Fig. 1, there are two main functions which are modulation identification and carrier recovery. $S W$ denotes a switch that opens or blocks the carrier recovery loop and $\mathrm{NCO}$ is a Numerically Controlled Oscillator outputting the signal used to cancel the offsets. As mentioned above, the Phase Error Detector (PED) and Low Pass Filter (LPF) are

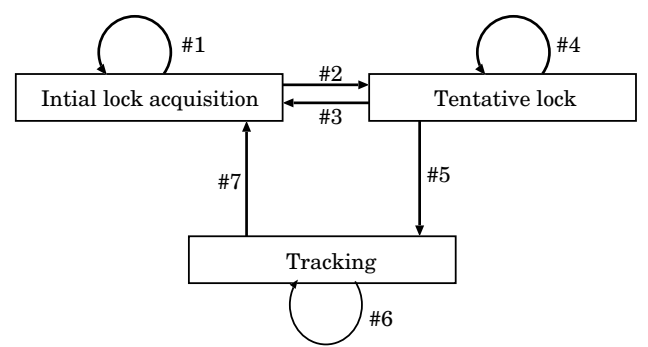

Fig. 2. State transition model of multimode PLL

designed to adapt to the transmitted modulation format. The multimode PLL has 3 states, which have key role to solve above conflicting problem. In fact, 3 states are termed 1) initial acquisition state, 2) tentative lock state and 3) tracking state as shown in Fig. 2. At the initial acquisition state, the modulation type is unknown, consequently, $S W$ is open and the received signals are input to the modulation identification block directly without the operation of PLL. In the modulation identification block, there is a bank of PLDs prepared for each modulation type, e.g. BPSK QPSK and 8PSK PLDs. PLD outputs the lock or non-lock signal from window by window. Here, size of window is denoted as $N$ symbols, and in this paper, $N=50$ [3]. Once any PLD outputs lock, the modulation type can be identified, and the state transits to tentative lock state (\#2 in Fig. 2). On the other hand, if lock can not be obtained by any PLD, namely this is non-MODID, the state is immovable (\#1 in Fig. 2). A role of tentative lock is a reconfirmation of the modulation identification result. In the tentative lock state, PLL is reconfigured with regard to the identified modulation type in the initial acquisition state and works to cancel phase and carrier offsets with closing $S W$. If the modulation identification results in tentative lock state and in initial acquisition state are the same, in that case, the state is changed to tracking state (\#5 in Fig. 2), otherwise, the state returns to the initial acquisition state. In fact, there is an arguable about the configuration of the state transition model, however, in this paper, we concentrate on the design of PLDs, hence, this paper presents only the procedures of the assumed state transition model. In the tracking state, PLL works in the same way as in tentative lock, and once non-lock is obtained in the PLD of the current modulation type identified in the initial acquisition and tentative lock states, the state returns to the initial acquisition state (\#7 in Fig. 2).

As is evident above descriptions, an important part of the modulation identification is PLD, hence, processing and design of PLDs are described in the following section.

\section{MODULATION IDENTIFICATION ALGORITHM}

To explain the modulation identification algorithm, at first, the logic and design of PLDs are explained. Next, the modulation identification logic utilizing the PLD outputs is presented.

\section{A. PLD logic and design}

The PLDs can be categorized as the Basic-PLD (BPLD) and the Sub-PLD (SPLD). The main role of BPLDs is carrier 


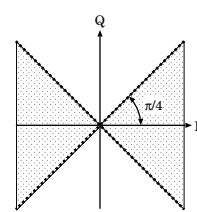

BPSK basic PLD

.

Lock

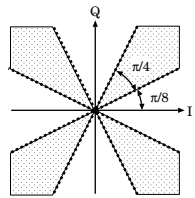

QPSK basic PLD

Non-Lock Area

....... Border line of Lock area

Fig. 3. Configurations of the Basic-PLDs

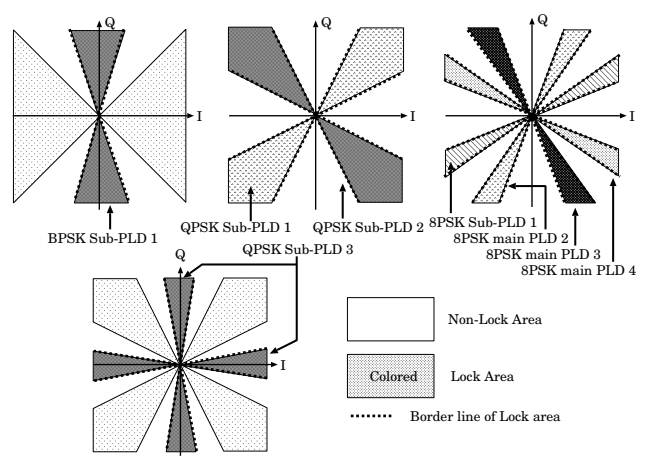

Fig. 4. Configurations of the Sub-PLDs

lock, on the other hand, the SPLDs are applied to improve the modulation identification performance. In fact, our studies have shown the performance of BPLDs in detail, and the efficiency of SPLDs. However, the theoretical analysis of the SPLDs seems to be lacking, hence, this paper intend to show the theoretical analysis and sub-optimized design of SPLD in section IV.

The BPLD of each modulation type is shown in Fig.3. Each PLD consists of the lock area and non-lock area, in addition, the design criterion is for the carrier lock detection. First motivation of this study is that the utilization of the BPLDs for the modulation identification, since the processing is similar between the lock detection and the modulation identification [3]. The behavior of BPLDs are very simple that each PLD counts the symbols lying in the lock area during a window. If the counting result $N_{C, P L D}$ is more than threshold $N_{T, P L D}$, PLD outputs 1 corresponding to lock, otherwise PLD outputs 0 corresponding to non-lock, where PLD indicates the type of PLD. In this paper, threshold of BPLDs $N_{T, P L D}$ is set at 27 according to [3]. In [3], the modulation identification algorithm employing only BPLDs was proposed and explained. However, the initial phase offset makes it difficult to identify the modulation identification, therefore, the SPLDs have been proposed. The configurations of SPLDs are shown in Fig. 4. SPLD contains the same components as in BPLD, and the behavior is also to count the symbols lying in lock area. Note the rule of lock regarding SPLDs is different from BPLD's rule. In BPSK SPLD 1 and QPSK SPLD 3 shown in Fig. 4, lock is obtained while $N_{C, P L D}<N_{T, P L D}$, on the other hand, the lock rule in other SPLDs is the same as BPLD's rule, e.g. $N_{C, P L D}>N_{T, P L D}$.

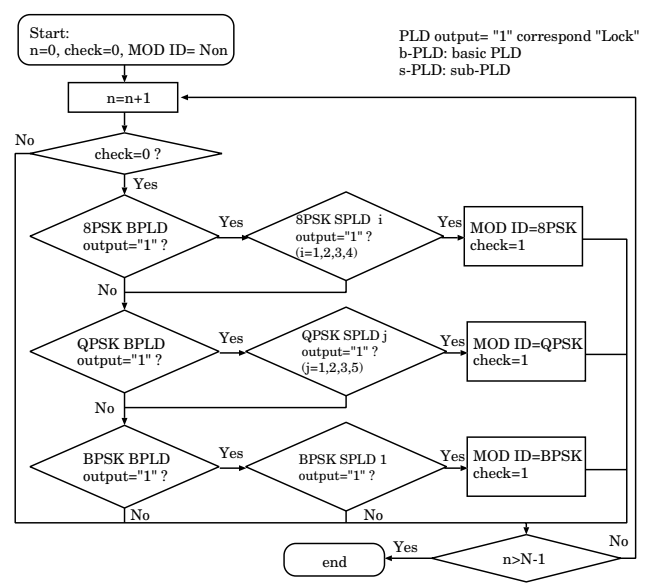

Fig. 5. Flowchart of modulation identification algorithm with Basic-PLDs and Sub-PLDs

\section{B. Modulation identification algorithm}

The detail of the modulation identification logic flowchart is shown in Fig 5. For a certain modulation type $M O D$, if $M O D-$ BPLD and all of the $M O D$-SPLDs output lock, this modulation type $M O D$ is the modulation identification result, and this state is denoted as "total lock". If several modulation types are candidates, e.g. "total lock" is obtained in several modulation types, the priority policy is used. In fact, the priority order is 8PSK, QPSK and BPSK as shown in Fig. 5, and this priority is based on an empirical rule [3].

\section{SUB-OPTIMIZED DESIGN OF PLD}

The criteria of sub-optimization is regarding the degradation level of throughput being denoted as $D_{L}$. Before presenting the optimization problem, the criteria is examined in detail as follows. In fact, this criteria is used in the comparison between the multimode PLL and the conventional scheme on ISDB-S in section V. As presented in section I, the conventional scheme employing pilot signal which can be said redundancy, i.e. ISDB-S employs 2 symbols for pilot signal and 203 symbols for information part. On the other hand, the multimode PLL does not have any redundancy, however, the modulation identification error $E R$ and the non-MODID $N R$ corresponds to the degradation level of the throughput. Finally, in the conventional scheme, $D_{L}=\frac{2}{255}$ and the multimode PLL, $D_{L}=E_{R}+N L . E R$ and $N R$ in mote carlo simulation are given by

$$
\begin{aligned}
& E R=M_{E R} / M_{\text {TOTAL }} \\
& N L=M_{N L} / M_{\text {TOTAL }}
\end{aligned}
$$

where $M_{T O T A L}, M_{E R}$ and $M_{N L}$ are a number of total trials, a number of the modulation identification error and a number of the non-lock, respectively. Theoretical analysis of $E R$ and $N L$ are described later. Based on $D_{L}$, the optimization problem is given by

$$
N_{T, S P L D, O P T}=\min _{N_{T, S P L D}} D_{L},
$$


where $N_{T, S P L D, O P T}$ is the optimized SPLD threshold. However, it is extremely difficult to obtain $N_{T, S P L D, O P T}$, therefore, we employ an alternative which is a sub-optimization in terms of minimizing $D_{L}$ under the determinate conditions. In order to explain the sub-optimization problem, we have to describe the reason why the SPLD is implemented in detail first. The modulation identification error is caused by the initial phase offset and the transmitted signal bias. The later case is that for example, if the QPSK signals are generated by only $C_{Q P S K}(n)=0$ or $C_{Q P S K}(n)=2$, the signal feature is completely the same as of BPSK completely, and modulation identification error occurs. In fact, the tentative lock state is countermeasure for this problem. An advantage of this state is utilization of multiple detections, namely, not only detection in initial acquisition, but also the detections in tentative lock are utilized. Inevitably, the modulation identification error due to bias can be avoided. An example of the former problem is shown in Fig. 6 where only BPLDs are employed and the modulation type is BPSK. When $\phi=\pi / 4$, most of the received signals lie in QPSK lock area, however, it is supposed that half of the received signals are in BPSK lock area. Therefore the modulation identification error occur easily. For this problem, SPLDs are employed, i.e. QPSK SPLD 1,2 and 8PSK SPLD 1,2,3,4. To explain the behavior of SPLD, we concentrate on the QPSK SPLD 1 and 2 which are a countermeasure against the modulation identification error, shown in Fig. 6, from BPSK to QPSK under initial phase offset. By implementing the QPSK SPLD1 and 2, the lock rule for QPSK is defined as shown in Fig. 5. On the other hand, the conventional lock rule, only applying the BPLDs, is shown as follows [3],

If $\left(N_{C, B-B P L D}>N_{T, B P L D}=27\right)$

LOCK: output 1

otherwise

Non-LOCK: output 0.

The additional conditions in proposed lock rule, shown in Fig. 5, $N_{C, \text { sub-QPSK1}}>N_{T, \text { sub-QPSK } 1} \& N_{C, \text { sub-QPSK } 2}>$ $N_{T, s u b-Q P S K 2}$, means that if there is a bias, lock rule detects the modulation type should not be QPSK. Therefore, even if the received signals modulated by BPSK have an initial phase offset, $\phi=\pi / 4$, such a modulation identification error type mentioned above can be avoided, and what is a important is setting $N_{T, S P L D}$.

Optimal solution of QPSK-SPLD design can be derived from minimizing sum of the theoretical formulations of $E R$ and $N R$ as shown in (5). However, the derivation of $E R$ is complicated, therefore, the total lock rate of QPSK, which is product of lock rates of QPSK BPLD and SPLD when the modulation type is BPSK in spite of $E R$. The sub-optimal problem can be mathematically formulated as follows:

$$
\begin{array}{r}
N_{T, S P L D, S O P T}=\min _{N_{T, S P L D}} P_{L, Q-P L D}\left(B, N_{T, S P L D}\right)+ \\
\left(1-P_{L, Q-P L D}\left(Q, N_{T, S P L D}\right)\right),
\end{array}
$$

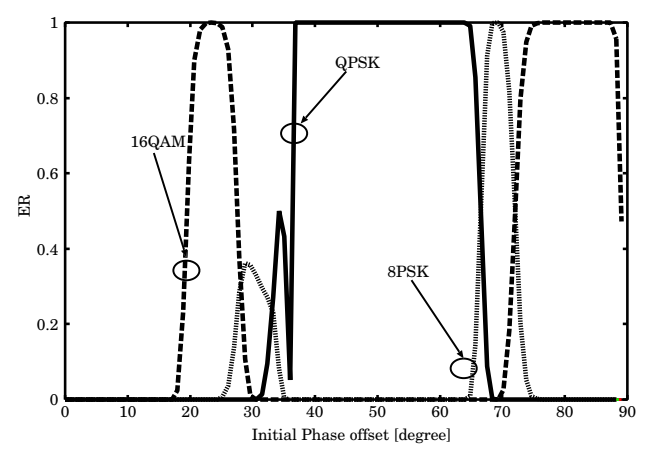

Fig. 6. Modulation Identification Error Rate vs Initial phase offset, Modulation: BPSK, Es/No=30dB, Carrier offset=0.001, Basic-PLDs

where $P_{L, Q-P L D}$ is the total lock rate of QPSK PLDs and $0 \leq N_{T, S P L D, S O P T} \leq N$. Here, the input parameter $B$ and $Q$ denote the modulation type, in this case, BPSK and QPSK, respectively. In addition, $P_{L, Q-P L D}$ is given by

$$
\begin{aligned}
P_{L, Q-P L D} & \left(M O D, N_{T, Q-S P L D 1}\right)=\int_{\phi} P_{L, Q-B P L D} \\
& \sum_{l_{N 2}} \sum_{N 1} P_{D}\left(l_{N 1}, l_{N 2}\right) p_{L, Q-S P L D 1}\left(N_{T, Q-S P L D 1}, l_{N 1}, \phi\right) \\
& p_{L, Q-S P L D 2}\left(N_{T, Q-S P L D 2}, l_{N 2}, \phi\right) d \phi,
\end{aligned}
$$

where $p_{L, P L D}, l_{N 1}$ and $l_{N 2}$ are lock probability of each PLD, the numbers of symbols assigned to $C_{Q P S K}=0,2$ and $C_{Q P S K}=$ 1,3 , respectively. Note, the lock probability $p_{L, P L D}$ can be obtained in the same way as [3], additionally, it is essential to consider the distributions of the symbols, i.e. $P_{D}\left(l_{N 1}, l_{N 2}\right)$ denoting the probability of such distribution, to obtains the total lock probability. Moreover, an approximation, which is $\Delta f=0$, is employed in lock probability. If we consider $\Delta f$, we should consider the order of the symbols, since the each symbol locates different position. In that case, a number of cases is $N$ !.

The comparison between the suboptimal solution (6) and optimal solution obtained by the monte carlo simulation is shown in Fig. 7 in terms of the QPSK and 8PSK SPLDs optimal thresholds. The difference between the suboptimal solution (6) and optimal solution is 1 at a maximum, therefore, we can achieve $N_{T, S P L D, S O P T}$ very close to $N_{T, Q-S P L D, O P T}$. Note, what we do not employ any SPLDs corresponds to $N_{T, Q-S P L D}=N$. However, in this analysis, there are 2 drawbacks. Firstly, the sub-optimal solution can not be formulated directly, namely, by calculating evaluation function (6) during $0 \leq N_{T, Q-S P S K} \leq N$, the optimal solution can be derived. Secondly, it is not proofed that one or zero existence of the minimal value during $0 \leq N_{T, Q-S P S K} \leq N$. This condition can be understood intuitively, and guarantee the sub-optimal solution (6).

\section{NUMERICAL EVALUATION}

This paper evaluates the multimode PLL in ISDB-S system where the adaptive modulation scheme is used. At first, the channel model and assumptions regarding ISDB-S standards, next, the comparison between proposal and the conventional system is shown in terms of $D_{L}$. 


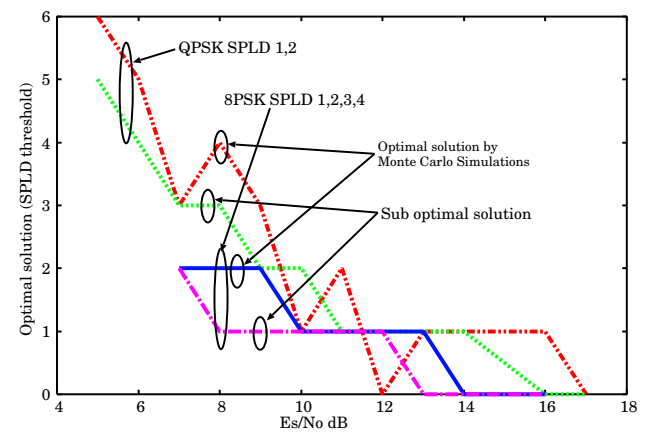

Fig. 7. Modulation Identification Error Rate vs Initial phase offset, Modulation: BPSK, Es $/ \mathrm{No}=30 \mathrm{~dB}$, Carrier offset=0.001, Basic-PLDs

\section{A. Channel Model}

The channel model is based on ISDB-S and ITU recommendations [4]-[7]. We assume the static channel model, i.e. the rain attenuation level is fixed during a window, since the fade slope is minuscule compared with the control speed in this system. By the [5]-[7], the estimated attenuation to be exceeded for other percentages of an average year, denoted $p_{R}$, is given by

$$
p_{R}=10^{11.628\left(-0.546+\sqrt{0.298+0.172 \log \left(0.12 A_{0.001} / A_{P}\right)}\right)}
$$

where,

$$
A_{0.001}=0.0173\left(R_{0.001}\right)^{1.205} \frac{5-0.075\left(\Phi_{L}-23\right)-h_{S}}{\sin \left(\theta_{A}\right)\left(1+L_{G} /\left(35 \exp \left(-0.015 R_{0.001}\right)\right)\right)}
$$

and $A_{P}$ is denoted as attenuation level. $p_{R}\left(A_{P}\right)$ indicates the cumulative distribution function (CDF) of attenuation level, in addition, by the numerical calculation method the probability distribution function (PDF) can be obtained. In the computer simulations shown as follows this PDF is used to obtain the statistic performances.

TABLE I

ISDB-S PARAMETERS [4]-[7]

\begin{tabular}{|c|c|}
\hline$R_{0.001}$ & 60 \\
\hline$h_{S}$ & $0 \mathrm{~km}$ \\
\hline$\theta_{A}$ & 38.3 degree \\
\hline$\Phi_{L}$ & $35 \mathrm{degree}$ \\
\hline Center frequency & $12 \mathrm{GHz}$ \\
\hline Band width & $34.5 \mathrm{M} \mathrm{Hz}$ \\
\hline Symbol rate & $28.86 \mathrm{M} \mathrm{symbol} / \mathrm{sec}$ \\
\hline Frame length & 205 symbols \\
\hline Number of pilot signals & 2 symbols \\
\hline Threshold of MOD change & $8 \mathrm{~dB}+$ margin and $11 \mathrm{~dB}+$ margin \\
\hline
\end{tabular}

\section{B. Degradation Level of Throughput}

Fig. 8 shows the degradation level of throughput $D_{L}$ regarding carrier offset. It can be seen that the multimode PLL can decrease $D_{L}$ while the margin level is more than 0dB. ISDB$\mathrm{S}$ has about $7 \mathrm{~dB}$ margin generally. Moreover, in the region where the carrier offset is less than $10^{-3}$, the influence due to the carrier offset can be cancelled.

In fact, the contribution to throughput of the multimode PLL is not significant, even the simplified technique is employed.

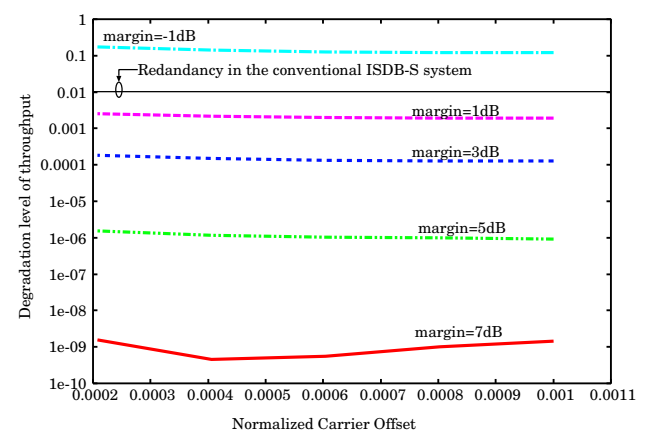

Fig. 8. $D_{L}$ vs carrier offset in ISDB-S employing adaptive modulation

However, it is confirmed that the modulation identification technique has a equivalent performance to the pilot signals in the conventional scheme. In addition, for more flexible communications, the modulation identification techniques are useful.

\section{CONCLUSION AND FUTURE SUBJECTS}

Multimode PLL, which can identify the modulation type and recover the carrier offset, is investigated in this paper. In particular, the main contributions are the derivation of the sub-optimal solutions of the SPLD and the evaluation of the multimode PLL on ISDB-S. Suboptimal solution is regarding to minimize $D_{L}$, in addition, a comparison of suboptimal solution and optimal solution presents the validity of suboptimal solution. Note, optimal solution is obtained by the monte carlo simulations. In the latter contribution, the multimode PLL is evaluated in terms of the throughput by comparing with the conventional scheme. It is confirmed that the multimode PLL has more advantage than conventional scheme. In addition, this paper is the first paper presenting the evaluations and efficiency of the modulation identification regarding throughput characteristic.

\section{REFERENCES}

[1] W. Wei and J. M. Mendel, "Maximum-Likelihood Classification for Digital Amplitude-Phase Modulations," IEEE Trans. Commun., vol. 48, pp. 189-193, Feb. 2000

[2] Chung-Yu Huang and A. Polydoros, "Likelihood Methods For MPSK Modulation Classification, " IEEE Trans. Commun., vol. 43, pp. 189193, Feb. 1995

[3] K. Umebayashi, R. H. Morelos-Zaragoza and R. Kohno, ”Method of Non-Data-Aided Carrier Recovery with Modulation Identification, " IEICE Trans., Fundamentals of Electronics, Communications and Computer Sciences. Vol.E87-A No.3 pp.656-665 2004

[4] Association of Radio Industries and Businesses "Transmission System for Digital Satellite Broadcasting," ARIB STD-B20.

[5] "ITU-R Recommendations. PN. 837-1: Characteristics of Precipitation for Propagation Modeling," ITU, ser. P, pt. 1, vol. 1997.

[6] "ITU-R Recommendations. 838: Specific Attenuation Model for Rain for Use in Prediction Methods," ITU, ser. P, pt. 1, vol. 1997.

[7] "ITU-R Recommendations. P. 618-5: Propagation Data and Prediction Methods Required for the Design of Earth-Space Telecommunication Systems," ITU, ser. P, pt. 2, vol. 1997. 